\title{
Eliminating subjective biases in judging the loudness of a $1-\mathrm{kHz}$ tone
}

\author{
E. C. POULTON and R. S. EDWARDS \\ Medical Research Council, Applied Psychology Unit, Cambridge CB2 2EF, England
}

and

T. J. FOWLER

Department of Engineering, Cambridge University, Cambridge, England

\begin{abstract}
It is possible to eliminate most of the known subjective biases that affect judgments of sensory magnitude using numbers. Experiments are described which do this, and which also investigate some of the biases. The least biased estimate for doubling the loudness of a $1-\mathrm{kHz}$ tone is found to be about $11.5 \mathrm{~dB}$. This value is still slightly affected by the logarithmic bias, although the bias could be eliminated. It is also affected by the stimulus equalizing bias, produced by the inequality between the finite range of loudnesses to which the ears are sensitive and the infinite range of numbers to which the loudnesses are matched. This last bias cannot be eliminated completely in direct magnitude estimation.
\end{abstract}

As a rough approximation, the biases that affect quantitative subjective judgments can be separated into two distinct kinds. First, there are the specific biases that are introduced by the experimenter's choice of the experimental details. In direct magnitude estimation, they include the choice of the exact stimuli, the responses, and the standard, if used. These specific experimenter biases are reviewed by Poulton (1968, Figure 1).

The present investigation is concerned with the second, more general kind of biases that are introduced by the observer in making his quantitative subjective judgments. They are illustrated in Figure 1 and listed on the left of Table 1 (Poulton, 1979). These more general subjective biases cannot be separated entirely from the specific experimenter biases, because experimental results depend upon both the experimenter's detailed choices and the observer's choices. But in studying subjective biases, the experimenter can attempt to minimize the specific biases that he himself introduces, or at least to hold them constant across comparisons.

The present paper describes an attempt to eliminate the subjective biases introduced by the observer. Two of the biases are investigated separately. The right side of Table 1 shows how the biases are dealt with. The loudness of a $1-\mathrm{kHz}$ tone is selected for study because it has been investigated experimentally more frequent-

\footnotetext{
We are grateful to $\mathrm{H}$. McRobert for permission to use his 1965 data, and to R. Patterson for calibrating the volume control for us. Financial support from the Medical Research Council to the first two authors is also gratefully acknowledged. Requests for reprints should be addressed to E. C. Poulton, M.R.C. Applied Psychology Unit, 15 Chaucer Road, Cambridge CB2 2EF, England.
}

ly than any other sensory dimension (Marks, 1974; Stevens, 1955).

\section{Range Biases}

The composite model of Figure 2 illustrates the relationship between the range biases, which are listed at the top of Table 1. The figure shows two sizes of response range plotted against two sizes of stimulus range.

In the centering bias of Figure $1 \mathrm{~A}$, the observer centers his range of responses upon the range of stimuli. In Figure 2, the centering bias determines the

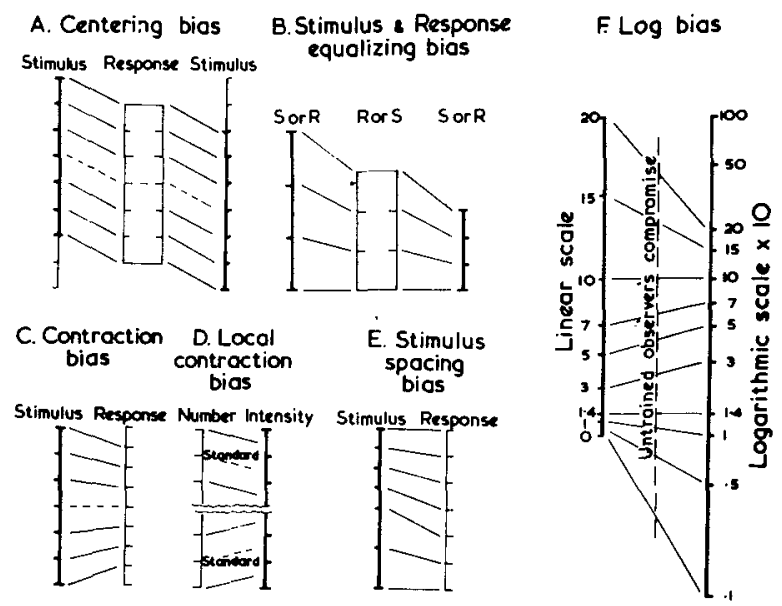

Figure 1. Models for the subjective biases in judging sensory magnitudes. Models $A, B$, and $C$ are concerned with the overall range, whereas Models $D, E$, and $F$ are concerned with the nonlinearities within the overall range ( $S=$ stimulus; $R=$ response). (From Poulton, 1979.) 


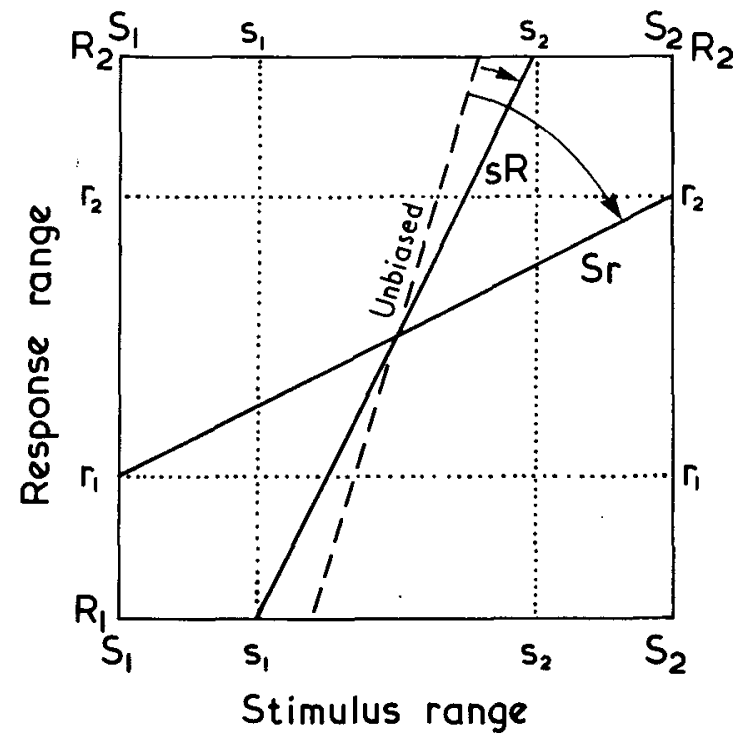

Figure 2. A composite model of the range biases. The centering bias determines the point at which the three straight lines cross. The contraction bjas reduces the slopes of the two solid lines compared with the slope of the theoretically unbiased dashed line. The difference in slope between the two solid lines reflects the stimulus and response equalizing biases. The slope is steeper with a small stimulus range (s) or a large response range (R), than with a large stimulus range ( $S$ ) or a small response range (r).

position of the point in the middle through which the sloping lines pass. Parducci (1963; Parducci \& Perrett, 1971) combines the centering bias with the stimulus-spacing bias of Figure $1 \mathrm{E}$ in his rangefrequency model. The model extends and modifies Helson's (1964) original adaptation-level model of the two biases. The top part of Table 1 shows that in the present investigation, the centering bias is avoided by using the very first judgments of groups of unpracticed observers.

The bias affecting the slopes of the lines in Figure 2 can be described most conveniently by dividing it into two parts. The contraction bias of Figure $1 \mathrm{C}$ is defined as the symmetrical part. It corresponds to Stevens and Greenbaum's (1966) regression effect. The remaining asymmetric part of the bias becomes the stimulus and response equalizing biases of Figure $1 \mathrm{~B}$.

In the stimulus and response equalizing biases of Figure 1B, the observer equates whatever the size of the stimulus range with whatever the size of his response range. The steeper solid line, labeled $S R$ in Figure 2, represents a small stimulus range paired with a large response range. The less steep solid line, labeled, $\mathrm{Sr}$, represents a large stimulus range paired with a small response range. Table 1 shows that in the present investigation the response equalizing bias is avoided by leaving the observer free to choose her own range of responses. The stimulus equalizing bias cannot be avoided completely in matching loudnesses and numbers, because the observer tends to use the same range of responses, either loudnesses or numbers, whatever the size of the range of stimuli (Poulton, 1979). The bias is partly excluded by discarding the most obviously biased points. But the remaining points must still be biased.

In the stimulus form of the contraction bias of Figure 1C, the observer underestimates large stimuli and differences between stimuli, and overestimates small stimuli and differences. In Figure 2, the dashed sloping line represents the theoretical function that is not affected by the contraction bias. The less steep slopes of the two solid lines are due partly to the contraction bias and partly to the stimulus and response equalizing biases. The contraction bias reduces the slopes because the contraction bias is larger with very large and very small stimuli than with stimuli of intermediate size.

Table 1 shows that the contraction bias is avoided in the present investigation by balancing magnitude adjustments using a volume control against numerical magnitude judgments, following Stevens and Greenbaum (1966). Figure 3 gives the data of McRobert, Bryan, and Tempest (1965) on the very first numerical judgments made by groups of unpracticed observers judging various ranges of intensity of



Sound pressure level in decibels

Figure 3. McRobert et al.'s (1965) very first multiple judgments of loudness of a 1-kHz tone. The open points on the abscissa represent the standard tone, which is always presented first and called 1.0. The open points on the dashed horizontal line for twice loudness show the theoretical slope of $10 \mathrm{~dB}$ for twice loudness. The filled points represent the geometric means of separate groups of between 9 and 19 undergraduates or university staff who are not practíced listeners. The sloping lines connect the geometric means to their standards. Lines passing to the left of the corresponding theoretical point for twice loudness indicate that less than $10 \mathrm{~dB}$ is required for twice loudness. Lines passing to the right indicate that more than $10 \mathrm{~dB}$ is required. 
Table 1

Subjective Biases in Judging Sensory Magnitudes

\begin{tabular}{|c|c|}
\hline Bias & How Dealt With \\
\hline \multicolumn{2}{|l|}{ 1. Range Biases } \\
\hline \multicolumn{2}{|l|}{ Centering } \\
\hline $\begin{array}{l}\text { The observer centers his range of responses upon } \\
\text { the range of stimuli }\end{array}$ & $\begin{array}{l}\text { Avoided by using the very first judgment of unpracticed } \\
\text { observers }\end{array}$ \\
\hline \multicolumn{2}{|l|}{ Stimulus Equalizing } \\
\hline $\begin{array}{l}\text { Whatever the size of the stimulus range, the observer } \\
\text { uses his full range of responses }\end{array}$ & $\begin{array}{l}\text { Partly excluded by discarding the most biased median } \\
\text { judgments }\end{array}$ \\
\hline \multicolumn{2}{|l|}{ Response Equalizing } \\
\hline $\begin{array}{l}\text { Whatever size response range the observer is given, he } \\
\text { distributes the responses over the stimulus range }\end{array}$ & $\begin{array}{l}\text { Avoided by leaving the observer free to choose the size } \\
\text { of his range of responses }\end{array}$ \\
\hline \multicolumn{2}{|l|}{ Contraction } \\
\hline $\begin{array}{l}\text { The observer underestimates large stimuli and differ- } \\
\text { ences between stimuli and overestimates small stimuli } \\
\text { and differences }\end{array}$ & $\begin{array}{l}\text { Avoided by balancing magnitude adjustments against } \\
\text { magnitude estimates }\end{array}$ \\
\hline $\begin{array}{l}\text { Once the observer knows the range of responses, he } \\
\text { selects a response too close to the middle of the range }\end{array}$ & $\begin{array}{l}\text { Investigated separately. Avoided by using a volume con- } \\
\text { trol with an unknown center and a numerical response } \\
\text { range that can extend from } 1.0 \text { to infinity without an } \\
\text { obvious middle value }\end{array}$ \\
\hline \multicolumn{2}{|l|}{ 2. Nonlinear Biases } \\
\hline \multicolumn{2}{|l|}{ Local Contraction } \\
\hline $\begin{array}{l}\text { The observer treats stimuli in small very high intensity } \\
\text { and very low intensity ranges as if they have less } \\
\text { extreme values }\end{array}$ & $\begin{array}{l}\text { Investigated separately. Avoided by not using a small } \\
\text { stimulus range with extreme values }\end{array}$ \\
\hline \multicolumn{2}{|l|}{ Stimulus Spacing } \\
\hline $\begin{array}{l}\text { The observer responds as if the stimuli are equally } \\
\text { spaced geometrically and equally probable }\end{array}$ & Avoided by using the very first judgment \\
\hline \multicolumn{2}{|l|}{ Logarithmic } \\
\hline $\begin{array}{l}\text { When there is a step change in the number of digits, } \\
\text { the observer compromises between a pure linear and } \\
\text { a pure logarithmic scale }\end{array}$ & $\begin{array}{l}\text { Avoided for the gain adjustments by using unpracticed } \\
\text { observers and single-digit numbers between } 1 \text { and } 10 \text {. } \\
\text { Bias-free data not available for the numerical judgments }\end{array}$ \\
\hline \multicolumn{2}{|l|}{ 3. Transfer Biases } \\
\hline Transfer from Previous Investigations & Investigated separately \\
\hline Transfer from Instructions and Demonstrations & \\
\hline $\begin{array}{l}\text { Transfer from Previous Stimuli } \\
\text { Transfer from Previous Judgments } \\
\text { Transfer from Previous Responses }\end{array}$ & $\begin{array}{l}\text { Avoided by using the very first judgment of unpracticed } \\
\text { observers with unbiased instructions and no demonstra- } \\
\text { tions }\end{array}$ \\
\hline
\end{tabular}

a $1-\mathrm{kHz}$ tone. The present investigation provides some of the corresponding data when the observer is given a volume control and is told to set it to an intensity a specified number of times greater than a standard intensity. Balancing the data obtained by the two methods eliminates the symmetrical contraction bias, because, by definition, the contraction biases of the two methods are equal and opposite in direction. The remaining assymmetries between loudness and numbers, produced by the difference in the sizes of the ranges, are, by definition, the stimulus and response equalizing biases.

As indicated at the bottom of Part 1 of Table 1, the contraction bias has a response form that occurs when the observer is given a range of responses with a known middle value. The observer selects a response too close to the middle of the range. In the present investigation, this means being familiar with the volume control. It is avoided by providing an unknown volume control for the very first judgment. The effect of seeing the volume control and its calibrations before using it is examined separately. In the experiment of Figure 3, McRobert et al. (1965) avoid this difficulty of a response range with an known middle value by asking for multiple numerical judgments. Here the responses range from 1.0 to infinity, and so have no obvious middle value.

\section{Nonlinear Biases}

The composite model of Figure 4 illustrates the relationships between the nonlinear biases, which are listed in Part 2 of Table 1. The figure shows a dashed function, whose center section is relatively free of bias, and two biased solid functions. Numerical responses are given on a linear scale on the ordinate. Stimulus intensity is given on a logarithmic scale on the abscissa.

The local contraction bias of Figure 1D is a nonlinearity that is described so far only for judgments of loudness (Poulton \& Stevens, 1955). The observer 


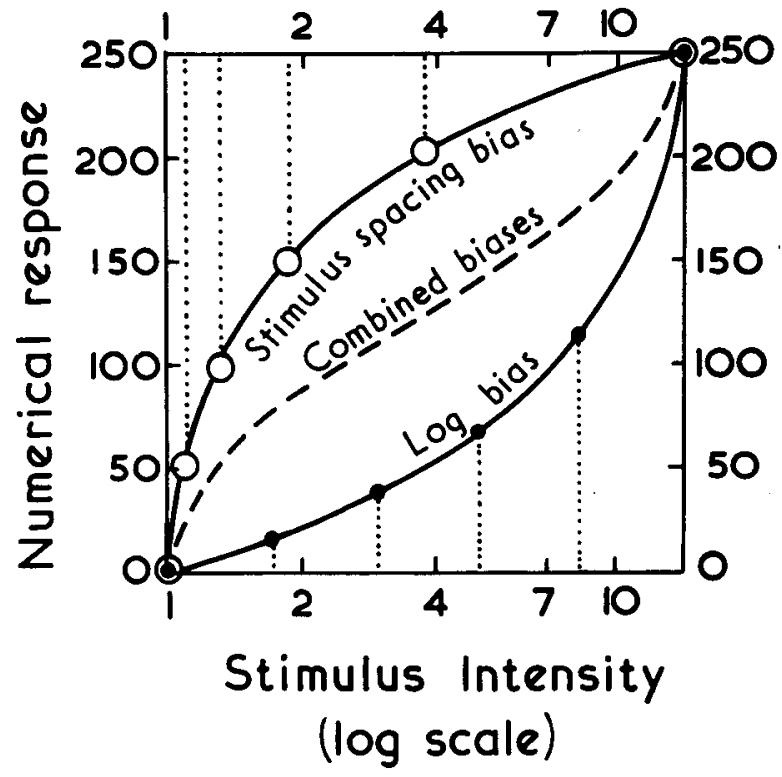

Figure 4. A composite model of the nonlinear biases. The lower solid function illustrates the logarithmic bias. The upper solid function illustrates the stimulus spacing bias in the theoretical absence of the logarithmic bias. The dashed function shows the stimulus spacing bias combined with and partly cancelling the logarithmic bias. The local contraction bias alters the slope of the dashed function at its top and bottom ends (see text).

treats acoustic stimuli in small very high-intensity and very low-intensity ranges as if they have less extreme values. For multiple judgments of high intensity, it reduces the slope of the dashed function in Figure 4 at the top end. But for fractional judgments of high intensity, it increases the slope. At the bottom end of the dashed function for judgments of low intensity, the local contraction bias has the reverse effect. It increases the slope for multiple judgments, but reduces the slope for fractional judgments. Part 2 of Table 1 shows that the local contraction bias is prevented by avoiding a small stimulus range with extreme values. This is one of the biases that is examined separately in the present investigation.

In the stimulus spacing bias of Figure 1E, the observer responds as if all the stimuli are equally spaced geometrically and equally probable. Equal geometric spacing means equal intervals on a logarithmic scale like that on the abscissa of Figure 4. The unfilled circles on the top function represent stimuli that are more closely spaced horizontally on the left of the figure than on the right. In the theoretical absence of the logarithmic bias, the observer allocates numbers to the stimuli as if they are equally spaced, judging each stimulus to be separated from the next by 50 . Thus, the solid function is steeper on the left than on the right. Table 1 shows that the stimulus spacing bias is avoided in the present investigation by using very first judgments.

The logarithmic bias of Figure 1F occurs whenever there is a step change in the number of digits used as stimuli or responses. To an observer who uses a logarithmic scale, there are as many numbers between 10 and 100 as there are between 1 and 10 , whereas to an observer who uses a linear scale, there are 10 times as many numbers between 10 and 100 as there are between 1 and 10. Untrained observers do not normally use numbers in either of these two ways. They compromise between the two pure scales, but use a scale that is nearer to logarithmic than to linear (Poulton, 1979).

In making numerical judgments, the logarithmic bias can affect the means, as well as the individual judgments. Since logarithmic and linear scales are related to each other nonlinearly, the observer's compromise scale is related nonlinearly to both scales. This biases both arithmetic means, which assume a linear scale, and geometric means, which assume a logarithmic scale. Only medians and nonparametric statistics are not affected by the nonlinearity produced by the logarithmic bias.

Figure IF shows that, compared with a linear scale, the logarithmic bias shrinks the upper part of the numerical scale. The lower solid function in Figure 4 illustrates the logarithmic bias in a series of judgments. The dashed function shows how the stimulus spacing bias can be made to cancel the logarithmic bias over the middle of the range of stimuli. Table 1 shows that the logarithmic bias is avoided by using unpracticed observers and single-digit numbers between 1 and 10.

\section{Range and Nonlinear Biases}

Figure 5 illustrates the distribution of the observer's responses that will account for all the range and nonlinear biases of Figure 1 and Table 1. The model makes no assumptions about the size of the range of stimuli selected by the experimenter. Any sized range will do. The symmetric distribution of responses produces the centering bias. Using almost the whole range of responses, whatever the size of the stimulus

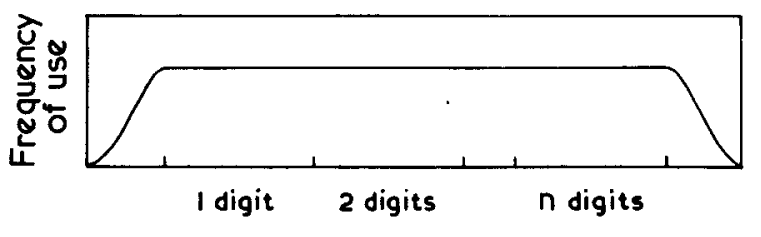

\section{Numerical response range}

Figure 5. The theoretical distribution of the observer's responses that will account for all the range and nonlinear biases listed in Table 1 (see text). The numerical scale on the abscissa represents the observers' responses in magnitude estimation. But the model works equally well when the experimenter presents the numbers and the observer adjusts the position of a volume control to match them. 
range, produces the stimulus and response equalizing biases. Avoiding the extreme responses, using instead less extreme values, produces the contraction biases. The flat top to the distribution indicates that the observer uses all except the extreme responses about equally often. This produces the stimulus spacing bias. Giving 1-digit, 2-digit, and in general n-digit numbers almost equal distances on the numerical scale of the abscissa produces the logarithmic bias.

\section{Transfer Biases}

The bottom part of Table 1 lists the transfer biases. They are avoided in the present investigation by using the very first judgments of unpracticed observers, with unbiased instructions and no demonstrations. The transfer bias from one trial to the next is one of the biases that is examined here.

\section{METHOD}

\section{Apparatus}

Two response buttons are mounted on a horizontal panel clamped to the side of a table. Pressing the white left-hand button produces a $1-\mathrm{kHz}$ tone of $39 \mathrm{~dB}$ re $20 \mu \mathrm{N} / \mathrm{m}^{2}$ in the headphones worn by the observer. Pressing the red right-hand button produces a $1-\mathrm{kHz}$ tone whose intensity depends upon the setting of a volume control.

The volume control is mounted on an instrument panel on the observer's right. The instrument panel rests on the edge of the table, facing away from the observer. the observer reaches the volume control by pushing her right hand under a black curtain which prevents her from seeing the instrument panel. She rests her forearm on the table with her elbow bent to about a right angle.

The knob of the volume control has a diameter of $5.5 \mathrm{~cm}$ and rotates through $315 \mathrm{deg}$. It has a circular $15-\mathrm{cm}$-diam disk of clear celluloid attached to its back, a semicircle of which is obscured by black paper. In Experiment 1, where the observer is shown the volume control, the black paper obscures half its calibrations. The observer can see only the numbers 1 through 13, arranged evenly round the semicircle of the dial that is visible.

The response characteristic of the volume control is illustrated in Figure 6 . It resembles fairly closely the "sone potentiometer" used by Stevens and Poulton (1956, Figure 1). But it deviates a little in the direction of a more linear relationship between the angle of rotation and decibels. Only one observer ever approaches the top intensity of $115 \mathrm{~dB}$. Before each trial, the volume control is set to the intensity of the standard tone of $39 \mathrm{~dB}$.

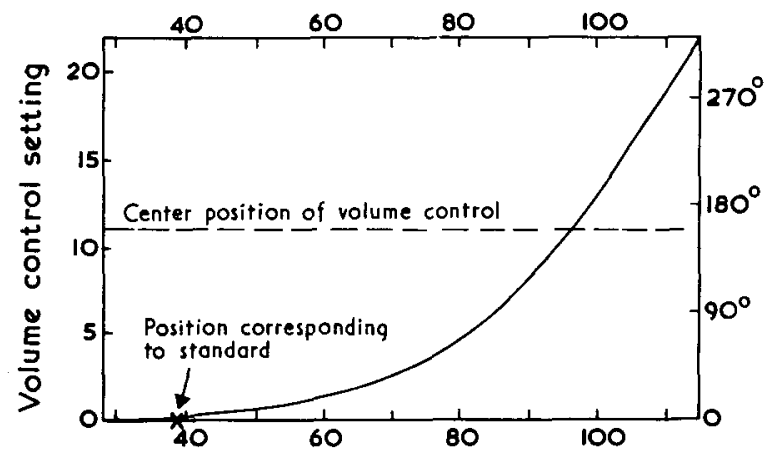

Sound pressure level in decibels

Figure 6. The relationship between the setting of the volume control and the sound pressure level in decibels. The numerical calibrations are given on the left ordinate, rotation in degrees is shown on the right ordinate.

\section{Procedure}

First, the observer is asked whether she has ever judged loudness before. In Experiment 1, she is then shown the volume control that she will be using. In Experiments 2 and 3, the volume control is always kept out of sight. When putting on the headphones, the observer is asked to make sure that they fit comfortably and to remove any hair that gets in the way.

The instructions run as follows: "When you press the left-hand white key, you will hear a soft tone. We can call its loudness one. Always press keys for a second or two. (The observer is instructed to press the key.) When you press the right-hand red key, the loudness of the tone depends upon how far up you have turned the volume control behind the black material. (The experimenter places the observer's right hand on the volume control.) I want you to adjust the volume control so that the loudness of the right-hand tone is twice $(10,30$, or 100 times) the loudness of the soft tone on the left. When you compare the two loudnesses, press the left key for a second or two, then pause, then press the right key for a second or two. You can compare the two loudnesses as often as you like, until you have set the volume control to what sounds like the correct loudness. When you are satisfied with your choice, please let me know. I suggest that you listen to both louder and softer tones from the volume control before you make your choice."

Any questions are answered by paraphrasing the instructions. If necessary, it is emphasized that the experimenter does not know appropriate setting of the volume control. This is what the experiment is designed to find out.

\section{Experimental Design}

In Experiments 1 and 2, each observer makes four adjustments to, respectively, twice, 10,30 , and 100 times the loudness of the standard. The order of the conditions is shown in Table 3 . In Experiment 1, there are 9 observers in each group. In Experiment 2 , there are 12 or 13 observers in each group, as indicated in Table 3.

In Experiment 3, each observer makes two adjustments, to 6 and 36 times the loudness of the standard, respectively. One group of 23 observers makes the two adjustments in this order. The other group of 22 observers makes the adjustments in the reverse order.

\section{Subjects}

Women members of the MRC Applied Psychology Unit subject panel served in Experiments 1 and 2, 36 and 49 of them, respectively. Their ages ranged from 19 to 55 years, median age 44 . They performed the experiment on arrival, before serving in an experiment on visual search. They were paid for their services. Four had served about 9 years previously in an experiment estimating the multiple loudness of a narrow band of noise centered on $1 \mathrm{kHz}$ (Poulton, 1969).

The 45 unpaid volunteers in Experiment 3 comprised 39 men and 6 women. They were caught as they passed through the foyer of the Cambridge University Engineering Department. Most were undergraduates of the department. Their ages ranged from 18 to 43 years, median age 22 . None said they had judged loudnesses previously. In all three experiments, the people were allocated to conditions in their order or appearance. The only exceptions were the four women who reported that they had judged loudness previously. They were distributed evenly between the main groups.

\section{Calculations and Statistical Tests}

In any experiment investigating an unknown, perhaps nonlinear, relationship between an independent and a dependent variable, medians and nonparametric statistical tests are the obvious choices. However, many experimenters calculate means in decibels, which are a logarithmic transformation of energy. Both medians and means are therefore given in Figures 7 and 8 for the gain adjustments.

For the numerical judgments, geometric means are used. Unfortunately, McRobert et al.'s (1965) data are no longer avalable for calculating medians. However, some indication of the likely difference between the geometric means and the medians of the very 


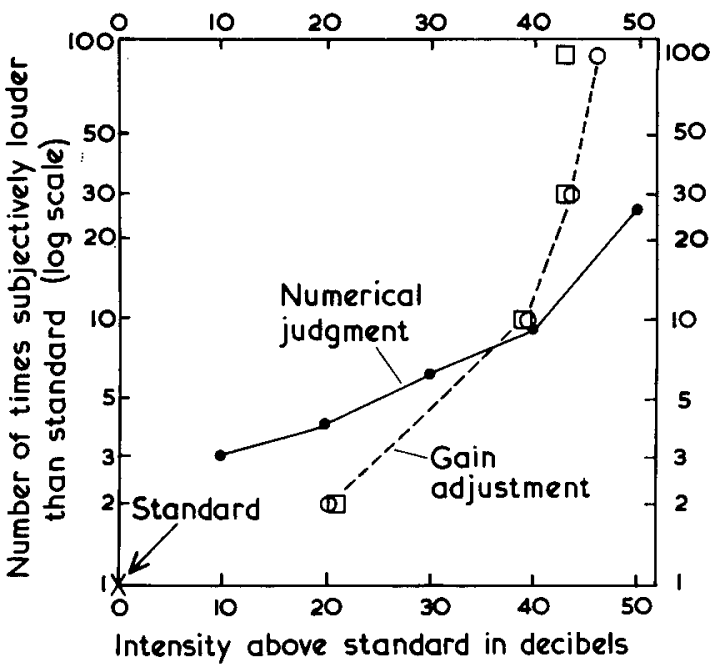

Figure 7. Very first multiple judgments of loudness by separate groups of unpracticed observers. The dashed line shows the pooled gain adjustments of Experiments 1 and 2. The unfilled squares represent the medians. The unfilled circles represent the corresponding means in decibels. Each median or mean is for a separate group of 21 or 22 women. The solid line shows the geometrically pooled geometric mean numerical judgments of Figure 3 (McRobert et al., 1965). Each filled circle represents a separate group of at least 50 undergraduates, except for the filled circle on the right, which is for only 24 undergraduates.

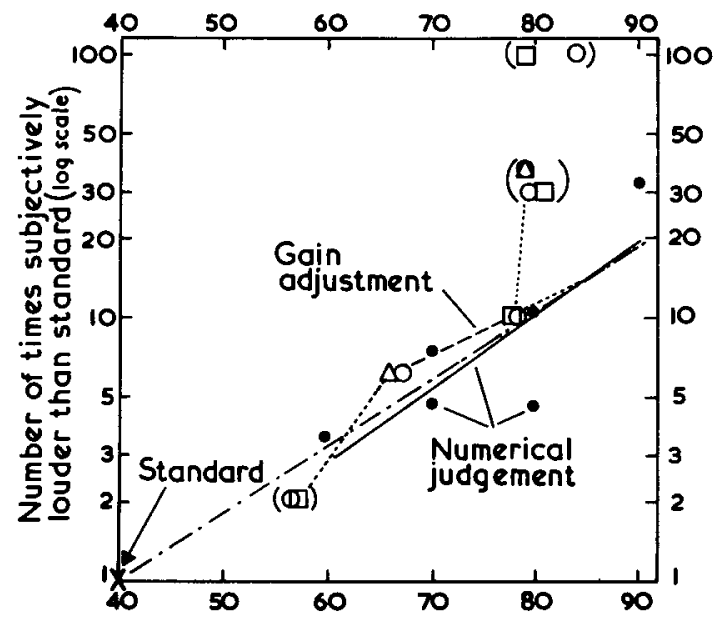

Sound pressure level in decibels

Figure 8. The least biased very first multiple judgments of loudness. The unfilied squares represent the medians of Experiment 2, without prior view of the volume control and without the two observers who judged loudness 9 years previously. The unfilled triangles represent the medians of Experiment 3. The unfilled circles show the corresponding means in decibels. All values are increased by $1.0 \mathrm{~dB}$ to superimpose the 39-dB standard upon the 40-dB standard used by McRobert et al. (1965) for the filled circles. The unflled points in brackets are greatly biased. The dashed line connects the two least biased medians. The solid line is fitted to the six filled circles which each represent the geometric mean of between 9 and 15 observers. The dotted and dashed line connects the origin to the point where the other two lines cross. first multiple numerical judgments of loudness can be obtained from the data of Poulton (1969), who used an octave band of noise centered on $1 \mathrm{kHz}$ and about 30 housewives in each group. Here geometric means of $2.3,7.2,8.0$, and 28.4 correspond to medians of $2,6,7$, and 20 , respectively. Thus, the logarithmic bias has a much greater effect upon very first judgments with geometric means greater than 10 than with geometric means less than 10 , as is to be expected. Only nonparametric statistical tests are used, Mann-Whitney U tests and Wilcoxon tests. All tests are twotailed.

\section{RESULTS}

\section{Pooled Biases}

Figure 7 gives the pooled results of Experiments 1 and 2 as open points. The open squares represent the medians of 21 or 22 very first adjustments. The open circles represent the corresponding means in decibels. The filled circles give the data from Figure 3 (McRobert et al., 1965). They represent the geometrically pooled geometric means of at least 50 very first numerical judgments, except for the filled circle on the right for only 24 judgments.

In all the experiments, the standard is shown at the origin with an intensity of $0 \mathrm{~dB}$ and a numerical value of 1 . The abscissa shows the increase in intensity above the standard. Thus, all the standards on the abscissa of Figure 3 are superimposed in Figure 7. The ordinate shows subjective loudness on a logarithmic scale.

\section{Contraction Bias}

The results in Figure 7 look reasonable orderly for very first judgments, because each point represents at least 21 people. The crossing of the broken function for the mean in decibels of the gain adjustments by the solid function for the geometric means of the numerical judgments, represents the contraction bias. It corresponds to Stevens and Greenbaum's (1966) regression effect. The crossing point is the only point that is by definition not affected by the contraction ,bias. It represents a loudness ratio of 8 for a difference of $37 \mathrm{~dB}$ from the standard. With the logarithmic scale on the ordinate, this means about $12 \mathrm{~dB}$ for twice or half loudness. It gives an exponent for sound amplitude of .5. (The slopes on a $\log \log$ plot like that of Figure 7 have to be doubled to give Stevens' exponents.)

The value of $12 \mathrm{~dB}$ is a little above Marks' (1974, Figure 1) modal value of about $11 \mathrm{~dB}$ reported in 23 experiments. It is a little further still above Stevens' (1955) composite value of about $10 \mathrm{~dB}$ for twice or half loudness, which was based upon all the previously published data at that time.

\section{Stimulus Equalizing Bias}

One unusual characteristic in Figure 7 is the almost vertical slope of the dashed function for the gain 
adjustments at the top on the right. For the medians represented by the unfilled squares, the top segment is vertical. The median gain adjustment is the same size whether the women are adjusting to 30 times the loudness of the standard or to 100 times the loudness. The women appear to be running out of responses. This is a ceiling effect imposed by the stimulus equalizing bias.

In obtaining gain adjustments, the experimenter has as infinite range of numerical stimulus ratios that extends from 1.0 to infinity, whereas the observer's response range of physical intensities is limited by what the ears can withstand or by what the observer is willing to inflict upon her ears. This corresponds to the less steep function $\mathrm{Sr}$ in Figure 2 for a large stimulus range paired to a small response range. In Figure 7 , for the gain adjustments, the stimuli are plotted vertically while the responses are plotted horizontally. This is the opposite of the labeling of the axes in Figure 2. Thus, the almost horizontal line $\mathrm{Sr}$ in Figure 2 corresponds to the almost vertical dashed line segments in Figure 7.

The solid function in Figure 7 for the numerical judgments must also be too steep. In obtaining numerical judgments, the sizes of the stimulus and response ranges are reversed. Here the experimenter has a finite range of stimulus intensities, while the observer has infinite range of numerical ratios. This corresponds to the steeper function SR in Figure 2 for a small stimulus range paired to a large response range. The bias increases the steepness of the solid function in Figure 7 for the numerical judgments. However, the right-hand segment of the solid line is not as steep as the final segment of the dashed line, owing to the contraction bias which has already been discussed.

\section{Volume Control Contraction Bias}

The response form of the contraction bias, at the bottom of Part 1 of Table 1, occurs when the range of responses has an obvious middle value and the observer knows the size of the range. In magnitude adjustments, this can happen when the observer knows the range of movement of the volume control. Before they start the experiment, the observers in Experiment 1 are shown the volume control and its calibrations in the arbitrary units of distance given on the left ordinate of Figure 6, while the observers in Experiment 2 are not. The effect upon the median difference from the standard of the very first adjustments is shown in Table 2.

The median differences from the standard of the groups of observers in Experiment 1, who see the volume control, are all larger than the corresponding medians of the groups of observers in Experiment 2, who do not. For the smallest angle of rotation, to increase loudness $\times 2$, the increase in decibels from 17 to 26 is reliable $(\mathrm{p}<.02)$.

A median of $26 \mathrm{~dB}$ above the standard of $39 \mathrm{~dB}$ makes a median intensity of $65 \mathrm{~dB}$. Figure 6 shows
Table 2

Effect of Seeing the Volume Control Before Making the Very First Adjustment

\begin{tabular}{ccccccc}
\hline & & & \multicolumn{4}{c}{$\begin{array}{c}\text { Median Increase in Decibels } \\
\text { Experi- } \\
\text { for SL Ratio }\end{array}$} \\
\cline { 5 - 7 } & $\begin{array}{c}\text { N per } \\
\text { Group }\end{array}$ & $\begin{array}{c}\text { Volume } \\
\text { Control }\end{array}$ & X2 & X10 & X30 & X100 \\
\hline 1 & 9 & Seen & $26^{*}$ & 39 & 44 & 53 \\
2 & 12 or 13 & Not Seen & $17^{*} \dagger$ & 38 & 39 & 40 \\
\hline
\end{tabular}

Note-SL $=$ subjective loudness

tReliably greater than $11 \mathrm{~dB}(p<.05)$

that $65 \mathrm{~dB}$ represents a rotation of only about $10 \%$ of the total range of rotation of the volume control. Thus, although seeing the volume control biases the median in the direction of the center of its range of movement, the median setting is nowhere near the middle of the range.

The largest median in Table 2 is $53 \mathrm{~dB}$, for an increase in loudness of $\times 100$. Adding the $39 \mathrm{~dB}$ of the standard gives a median intensity of $92 \mathrm{~dB}$. Figure 5 shows that even this represents a rotation of only $40 \%$ of the total range of rotation of the volume control. Thus, in spite of seeing the volume control, all the medians are on the lower side of the center of its range of rotation, like the intensity corresponding to the standard.

\section{Local Contraction Bias}

The local contraction bias is described only for acoustic stimuli in small very high- and very low-intensity ranges. In Table 2 , it applies only to the gain adjustment $\times 2$. The local contraction bias increases the number of decibels required to double the intensity of a soft sound, compared with the number required to double the intenstiy of a sound of moderate intensity. Here the bias is in the same direction as the contraction bias, which accounts for the overestimation of small differences. For the observers in Experiment 1, the bias is also in the same direction as the volume control contraction bias. Thus, the results of Experiment 1 need to be excluded in attempting to isolate the local contraction bias.

Table 2 shows that, in Experiment 2, twice the loudness of the standard of $39 \mathrm{~dB}$ requires a median increase in intensity of $17 \mathrm{~dB}$. The value of $17 \mathrm{~dB}$ is reliably $(p<.05)$ larger than Stevens' $(1955)$ composite value of $10 \mathrm{~dB}$ for twice or half the loudness of tones and Marks' (1974) modal value of $11 \mathrm{~dB}$ for twice or half loudness. The relatively large value of $17 \mathrm{~dB}$ is increased by the contraction bias, which increases all judgments of small differences like twice or half loudness, as well as by the local contraction bias. However, if anything, Stevens' and Marks' values of 10 and $11 \mathrm{~dB}$ are also likely to be slightly increased by the contraction bias.

Stevens' (1955) composite value of $10 \mathrm{~dB}$ is based largely upon judgments of halving or doubling. Thus, they correspond most closely to the points on the 
extreme left of the two functions of Figure 7. Connecting these two points to the standard at the origin shows that the gain adjustments have the smaller slope, and hence require more decibels for doubling and halving than do the numerical judgments. Stevens includes in his data about twice as many gain adjustments as numerical judgments. Thus, if anything, his composite value of $10 \mathrm{~dB}$ should be slightly too large.

This argument applies also to the seven experiments on halving or doubling used by Marks (1974). Here, again, there are about twice as many gain adjustments as numerical judgments. However, Marks' modal of value of $11 \mathrm{~dB}$ is based predominantly upon the slopes of complete psychophysical functions like those of Figure 7. Here the figure shows that it is the numerical judgments that have the smaller slope, and hence require more decibels for doubling and halving than do the gain adjustments. Marks includes about three times as many complete psychophysical functions derived from numerical judgments as from gain adjustments. The predominance of complete psychophysical functions derived from numerical judgments, which require more decibels for doubling and halving, means that his modal value of $11 \mathrm{~dB}$ should be slightly too large, like Stevens' composite value of $10 \mathrm{~dB}$.

In comparing the $17 \mathrm{~dB}$ required for doubling loudness of Experiment 2 in Table 2 with the values of 10 and $11 \mathrm{~dB}$ given by Stevens and by Marks, all three values are therefore likely to be exaggerated by the contraction bias, although the $17-\mathrm{dB}$ value may be the most exaggerated. Thus, much of the reliable difference between the $17 \mathrm{~dB}$ and the values of 10 and $11 \mathrm{~dB}$ is likely to be due to the local contraction bias, although some of it is probably due to the contraction bias.

\section{Bias by Transfer}

Table 3 shows the effect of prior gain adjustments in Experiment 2. The orders of the four trials performed by the four groups of observers are shown in the four columns on the left. The median differences from the standard in the gain adjustments are shown in the four columns on the right.

The first row of Table 3 on the right shows the median differences from the standard of the very first gain adjustments. They correspond to the values in row 2 of Table 2 . The medians for $\times 10, \times 30$, and $\times 100$ are all about the same, 38 to $40 \mathrm{~dB}$. The subsequent rows of the table show that the medians are more spread out in the later trials. The medians for $\times 10$ fall reliably from $38 \mathrm{~dB}$ in the first trial to $30 \mathrm{~dB}$ in the second trial $(p<.002)$, and from then on remain at $30 \mathrm{~dB}$ or below. The medians for $\times 30$ oscillate in Trials 2 to 4 around the $39 \mathrm{~dB}$ of Trial 1 , while the medians for $\times 100$ increase reliably from $40 \mathrm{~dB}$ in the first trial to $49 \mathrm{~dB}$ in the second trial $(p<.05)$ and then increase gradually to $53 \mathrm{~dB}$ in the fourth trial. The spreading out reflects the influence of the previous judgments in the absence of any
Table 3

Effect of Transfer from Preceding Trials in Experiment 2

\begin{tabular}{|c|c|c|c|c|c|c|c|c|}
\hline \multirow{2}{*}{$\begin{array}{l}\text { Order } \\
\text { of Con- } \\
\text { ditions }\end{array}$} & \multicolumn{4}{|c|}{$\mathrm{N}$ of Observers } & \multicolumn{4}{|c|}{$\begin{array}{l}\text { Median Increase in } \\
\text { Decibels for } \\
\text { SL Ratio }\end{array}$} \\
\hline & 13 & 12 & 12 & 12 & $\mathrm{X} 2$ & $\mathbf{X} 10$ & $\times 30$ & $\mathrm{X} 100$ \\
\hline $\begin{array}{l}1 \\
2 \\
3 \\
4\end{array}$ & $\begin{array}{r}\mathrm{X} 2 \\
\mathrm{X} 10 \\
\mathrm{X} 30 \\
\mathbf{X 1 0 0}\end{array}$ & $\begin{array}{r}X 10 \\
\times 100 \\
\times 2 \\
\times 30\end{array}$ & $\begin{array}{r}\mathrm{X} 30 \\
\mathrm{X} 2 \\
\mathbf{X 1 0 0} \\
\mathrm{X} 10\end{array}$ & $\begin{array}{r}\mathrm{X} 100 \\
\times 30 \\
\times 10 \\
\times 2\end{array}$ & $\begin{array}{l}17 \\
19 \\
17 \\
18\end{array}$ & $\begin{array}{l}38 * \\
30 \\
24 \\
30\end{array}$ & $\begin{array}{l}39 \\
30 \\
40 \\
37\end{array}$ & $\begin{array}{l}40 \dagger \\
49 \dagger \\
50 \\
53\end{array}$ \\
\hline
\end{tabular}

Note-Volume control not seen. $S L=$ subjective loudness. * $X 10$ first largest in column $(p<.05$ or better)

$+X 100$ first smaller than $X 100$ second $(p<.05)$

external knowledge of results. It shows how the stimulus spacing bias of Figure $1 \mathrm{E}$ operates over a series of magnitude judgments.

The spreading out does not apply to twice loudness. Here Table 3 shows that the median difference from the standard remains throughout at about $17 \mathrm{~dB}$. Most of the early investigations of judgments of loudness stick to ratios of twice and half loudness (Stevens, 1955). The results in Table 3 suggest that this avoids the transfer that may bias the larger ratios of loudness when they are included in a withinsubjects design.

Four women judged the loudness of a narrow band of noise centered on $1 \mathrm{kHz}$ about 9 years before serving in the present experiments. The standard was about $65 \mathrm{~dB}$, and the variables ranged from 70 to $100 \mathrm{~dB}$ (Poulton, 1969). One woman served in each of the four composite groups represented by the open points in Figure 7. Two of the women made adjustments above the median of their group, while two made adjustments below the median. Their results bear no obvious relationship to their experience 9 years previously.

\section{Logarithmic Bias}

The logarithmic bias in the use of numbers produces a number scale that is intermediate between linear and logarithmic, as illustrated in Figure 1F. On the log log plot of Figure 7, it produces functions that are concave downward, though not as concave downward as a linear number scale. The direction of the bias depends upon whether it is believed that the observers should be using a linear or a logarithmic number scale. Figure 1F shows that all three number scales are fairly similar in the region between 2 and 10 . Thus, of the unfilled points for the gain adjustments in Figure 7 , only those for $\times 30$ and $\times 100$ are likely to be much affected by the logarithmic bias.

Of the filled points for the numerical judgments, the point at the top on the right with a geometric mean of 25.9 is likely to be the most biased. But all the geometric means are likely to be higher than the corresponding medians. Estimating from the data of Poulton (1969), given in the section on calculations, the geometric means of $3.1,4.0,6.3,9.1$, and 25.9 
in Figure 7 probably represent medians of $2,3,5,8$, and 20 , respectively.

\section{Minimum Bias}

Figure 8 illustrates an attempt to eliminate as many biases as possible. The six filled circles are the geometric means of the very first numerical judgments illustrated in Figure 3 for the standard of $40 \mathrm{~dB}$. The unfilled squares represent the median first gain adjustments of Experiment 2. The unfilled triangles represent the corresponding medians from Experiment 3. These are the two experiments in which the observer's very first judgment is not biased by seeing the volume control before she or he starts. Not included are the adjustments of the two observers who judged loudness about 9 years previously.

The unfilled circles in Figure 8 represent the corresponding means in decibels of Experiments 2 and 3. The means correspond to the geometric means represented by the filled circles for the numerical judgments. Since, in Experiments 2 and 3, the standard turns out to be $39 \mathrm{~dB}$, instead of the $40 \mathrm{~dB}$ shown in the figure, all the decibel values of Experiments 2 and 3 are increased by 1.0 to make them comparable to the decibel values of the filled circles.

The points in Figure 8 that are obviously biased are shown in brackets. The two unfilled squares and the unfilled triangle all at the top, representing the median loudness ratios of 30,36 , and 100 , lie almost vertically above the unfilled square for the median loudness ratio of 10 . Thus they are greatly affected by the stimulus equalizing bias. The unfilled square for the median loudness ratio of 2 is affected by the local contraction bias. This leaves unbracketed only the unfilled triangle and square for the median loudness ratios of 6 and 10 .

Transfer from previous judgments of loudness is excluded by using only the very first judgments of unpracticed observers. The two women who served in Experiment 2 after serving 9 years previously in an experiment on numerical judgments of loudness are also excluded.

The unfilled triangle and square in Figure 8 for the median very first gain adjustments to 6 and 10 times loudness are not likely to be affected by the logarithmic bias because the ratios lie within or at the limits of the range of single-digit numbers. Also, they are probably not much affected by the contraction bias, because they lie near the middle of the range of sound intensities commonly met in everyday life, of from 60 to $80 \mathrm{~dB}$. Thus they are relatively unbiased. When the midpoint of the dashed line connecting the two medians is joined to the standard at the origin of the figure, it has a slope that gives about $11 \mathrm{~dB}$ for twice or half loudness. This is about Marks' (1974, Figure 1) modal value reported in 23 experiments, which has already been referred to.

The solid line in Figure 8 is fitted to the six filled circles, which represent the geometric means of the very first numerical judgments. When the midpoint of the solid line at $75 \mathrm{~dB}$ and 7.6 times loudness is joined to the standard at the origin of the figure, it has a slope that gives about $12 \mathrm{~dB}$ for twice or half loudness.

As already pointed out, the slope would be smaller if the less biased medians were available for use. The pairs of geometric means for 70 and $80 \mathrm{~dB}$ can each be combined geometrically. This gives the four geometric means for the numerical judgments at 60,70 , 80 , and $90 \mathrm{~dB}$, respectively, or $3.4,6.2,7.5$, and 32.5. The data of Poulton (1969), given in the section on calculations, suggest that the corresponding medians are most probably, $2,5,6$, and 20 . These four estimated medians can be plotted on a graph like that of Figure 8. When a straight line is fitted to the four points, its midpoint at $75 \mathrm{~dB}$ represents 5.9 times loudness. Joining this midpoint to the standard and at the origin gives a slope that corresponds to about $14 \mathrm{~dB}$ for twice or half loudness. It suggests that the value of about $12 \mathrm{~dB}$ obtained from the geometric means is about $2 \mathrm{~dB}$ too small.

To remove the contraction bias, the average for twice or half loudness of about $12 \mathrm{~dB}$ obtained from the geometric mean numerical judgments can be balanced against the average of about $11 \mathrm{~dB}$ obtained from the least biased median gain adjustments. The combined average gives about $11.5 \mathrm{~dB}$ for twice or half loudness. The dashed and dotted line in Figure 7 connects the origin of the figure to the point where the extrapolated dashed line crosses the solid line. It also gives about $11.5 \mathrm{~dB}$ for twice or half loudness.

Unfortunately, the filled circles representing the geometric mean numerical judgments are influenced by the logarithmic bias. Thus, balancing the gain adjustments against the magnitude judgments removes the contraction bias at the expense of introducing some logarithmic bias. However, the principal bias of the dashed and dotted line is likely to be the residual stimulus equalizing bias, which is produced by the unequal sizes of the range of intensities and the range of numbers that are available. As already indicated, the stimulus equalizing bias increases the steepness of any function relating subjective magnitude in numbers to physical intensity on a $\log \log$ plot like that of Figure 8, when the range of numbers available is infinite but the range of intensities is finite. The bias cannot be eliminated completely in an experiment on direct magnitude estimation.

\section{DISCUSSION OF PREVIOUS WORK}

Stevens (1956) is the first experimenter to publish a paper on the biases in direct magnitude estimation. Of the general biases introduced by the observer, he describes the response equalizing bias of Figure 1B the local contraction bias of Figure 1D, the stimulus 
spacing bias of Figure $1 \mathrm{E}$, the logarithmic bias of Figure 1F, and bias from transfer.

Stevens (1956) also discusses some of the specific biases introduced by the experimenter's choice of the experimental details. He compares magnitude estimates using standards of different intensities and at different positions in the range of variables, and with different numerical values or moduli. He also reports some results without a standard. He suggests (p. 14) how the standard could perhaps be changed during the course of an investigation, by using a modulus for the new standard consistent with the previous judgments. He concludes that his least biased results are consistent with his (Stevens, 1955) composite value of $10 \mathrm{~dB}$ for twice or half loudness.

Hellman and Zwislocki (1961) follow up Stevens' work on the specific biases introduced by the experimenter's choice of the experimental details, but they do not deal explicitly with the general biases introduced by the observer. They combine the results from two separate ranges of intensities, a more intense range of from 20 to $100 \mathrm{~dB}$ SL using a standard of $70 \mathrm{~dB}$, called 10 , and a less intense range of from threshold to $60 \mathrm{~dB}$ SL using a standard of $40 \mathrm{~dB}$, called 1. Their final psychophysical function for a $1-\mathrm{kHz}$ tone gives about $11 \mathrm{~dB}$ for twice or half loudness over most of the range of usable intensity. By measuring intensity from each person's auditory threshold, using sound level (SL) instead of the conventional sound pressure level (SPL), they are able to extend the psychophysical function for loudness down to $4 \mathrm{~dB}$ above threshold. Here the psychophysical function becomes steeper because, of course, on a $\log \log$ plot, the slope has to increase to infinity at the threshold (Poulton, 1968, Figure 1B).

Both Hellman and Zwislocki (1961) and Stevens (1956) appear to deal with the biases intuitively, rejecting experimental procedures because they give unacceptable results, rather than because they are known to introduce bias. This is presumably the only possible way to proceed before the biases have been described and studied. But it is probably not necessary to use this intuitive approach today, now that the biases are better known.

In a later review, Stevens (1971) discusses the three remaining general subjective biases of Figure 1 introduced by the observer: the centering bias of Figure 1A, which Stevens (p. 428) eliminates by modulus equalization; the stimulus equalizing bias of Figure 1B described by Jones and Woskow (1962); and the contraction bias of Figure 1C described by Stevens and Poulton (1956) and subsequently investigated more fully by Stevens and Greenbaum (1966). In 1971, Stevens proposes (p. 429) about $9 \mathrm{~dB}$ for twice or half loudness.

Warren $(1970,1973)$ uses the very first fractional numerical judgments between 100 and 0 of separate groups of students for each intensity of the variable, holding the standard intensity constant. He avoids the response form of the contraction bias listed at the bottom of Part 1 of Table 1, selecting a response too close to the middle of the range or responses. This is done by using the judgments only of the variable stimulus which gives a median numerical estimate of 50. This variable is, on average, at the center of the range of responses between 100 and 0 , where the response contraction bias is equal and opposite in the up and down directions. Using this method, Warren finds that a reduction of $6 \mathrm{~dB}$ produces half loudness as predicted by the inverse square law for the reception of energy at a distance (Warren, Sersen, \& Pores, 1958).

But, unfortunately, this technique fails to avoid the stimulus form of the contraction bias, overestimating the size of small stimulus differences. In judging loudness, a difference of $6 \mathrm{~dB}$ is a relatively small difference which people tend to overestimate, like the difference of $10 \mathrm{~dB}$ represented by the filled circle on the left of Figure 7. Thus, $6 \mathrm{~dB}$ gives too steep a slope on a log log plot, and hence too few decibels for half or twice loudness.

\section{REFERENCES}

Hellman, R. P., \& Zwislocki, J. Some factors affecting the estimation of loudness. Journal of the Acoustical Society of America, 1961, 33, 687-694.

Helson, H. Adaptation-level theory. New York: Harper \& Row, 1964.

Jones, F. N., \& Woskow, M. J. On the relationship between estimates of magnitude of loudness and pitch. American Journal of Psychology, 1962, 75, 669-671.

McRobert, H., Bryan, M. E., \& Tempest, W. Magnitude estimation of loudness. Journal of Sound and Vibration, 1965, 2,391-401.

Marks, L. On scales of sensation: Prolegomena to any future psychophysics that will be able to come forth as science. Perception \& Psychophysics, 1974, 16, 368-376.

Paruucci, A. Range-frequency compromise in judgment. Psychological Monographs, 1963, 77(2, Whole No. 565).

Parducci, A., \& Perretr, L. F. Category rating scales: Effects of relative spacing and frequency of stimulus values. Journal of Experimental Psychology Monograph, 1971, 89, 427-452.

Poulton, E. C. The new psychophysics: Six models for magnitude estimation. Psychological Bulletin, 1968, 69, 1-19.

Poulton, E. C. Choice of first variables for single and repeated multiple estimates of loudness. Journal of Experimental Psychology, 1969, 80, 249-253.

Poulton, E. C. Models for the biases in judging sensory magnitudes. Psychological Bulletin, 1979, 86, 777-803.

Poulton, E. C., \& Stevens, S. S. On the halving and doubling of the loudness of white noise. Journal of the Acoustical Society of America, 1955, 27, 329-331.

STEVENS, S. S. The measurement of loudness. Journal of the Acoustical Society of America, 1955, 27, 815-829.

STEVENS, S. S. The direct estimation of sensory magnitudesloudness. American Journal of Psychology, 1956, 79, 1-25.

Stevens, S. S. Issues in psychophysical measurement. Psychological Review, 1971, 78, 426-450.

Stevens, S. S., \& Greenbaum, H. B. Regression effect in 
psychophysical judgment. Perception \& Psychophysics, 1966, 1, 439-446.

Stevens, S. S., \& Poulton, E. C. The estimation of loudness by unpracticed observers. Journal of Experimental Psychology, 1956, 51, 71-78.

Warren, R. M. Elimination of biases in loudness judgments for tones. Journal of the Acoustical Society of America, 1970, 48, 1397-1403.
WARren, R. M. Quantification of loudness. American Journal of Psychology, 1973, 86, 807-825.

Warren, R. M., Sersen, E., \& Pores, E. A basis for loudness judgments. American Journal of Psychology, 1958, 71, 700-709.

(Received for publication January 2, 1979; revision accepted November $7,1979$. 